\title{
Harnessing adipose-derived stem cells to release specialized secretome for the treatment of hepatitis $B$
}

\author{
HEE JU KIM ${ }^{1}$, OK-HEE KIM ${ }^{1,2}$, HA-EUN HONG ${ }^{1,2}$, SANG CHUL LEE ${ }^{2,3}$ and SAY-JUNE KIM ${ }^{1,2}$ \\ ${ }^{1}$ Department of Surgery, Seoul St. Mary's Hospital, College of Medicine, The Catholic University of Korea, \\ Seoul 06591; ${ }^{2}$ Catholic Central Laboratory of Surgery, Institute of Biomedical Industry, College of Medicine, \\ The Catholic University of Korea; ${ }^{3}$ Department of Surgery, Daejeon St. Mary's Hospital, College of Medicine, \\ The Catholic University of Korea, Daejeon 34943, Republic of Korea
}

Received December 27, 2019; Accepted December 9, 2020

DOI: $10.3892 /$ ijmm.2021.4848

\begin{abstract}
Mesenchymal stem cells (MSCs) have the function of repairing damaged tissue, which is known to be mediated by the secretome, the collection of secretory materials shed from MSCs. Adjusting the culture conditions of MSCs can lead to a significant difference in the composition of the secretome. It was hypothesized that pre-sensitization of MSCs with specific disease-causing agents could harness MSCs to release the therapeutic materials specialized for the disease. To validate this hypothesis, the present study aimed to generate a 'disease-specific secretome' for hepatitis caused by hepatitis B virus using hepatitis BX antigen ( $\mathrm{HBx})$ as a disease-causing material. Secretary materials (HBx-IS) were collected following the stimulation of adipose-derived stem cells (ASCs) with 100-fold diluted culture media of AML12 hepatocytes that had been transfected with pcDNA-HBx for $24 \mathrm{~h}$. An animal model of hepatitis B was generated by injecting $\mathrm{HBx}$ into mice, and the mice were subsequently intravenously administered a control secretome (CS) or HBx-IS. Compared with the CS injection, the HBx-IS injection significantly reduced the serum levels of interleukin- 6 and tumor necrosis factor- $\alpha$ (pro-inflammatory cytokines). Western blot analysis and immunohistochemistry of the liver specimens revealed that the HBx-IS injection led to a higher expression of liver regeneration-related markers, including hepatocyte growth factor and proliferating cell nuclear antigen, a lower expression of pro-apoptotic markers, such as cleaved caspase 3 and Bim in mouse livers, and a lower expression of pro-inflammatory markers (F4/80 and CD68) compared to the CS injection. HBx-IS exhibited higher liver regenerative, anti-inflammatory
\end{abstract}

Correspondence to: Professor Say-June Kim, Department of Surgery, Seoul St. Mary's Hospital, College of Medicine, The Catholic University of Korea, 222 Banpo-daero, Seocho-gu, Seoul 06591, Republic of Korea

E-mail: sayjunekim@gmail.com

Key words: adipose-derived stem cell, hepatitis B virus, hepatitis BX antigen, mesenchymal stem cell, secretome and anti-apoptotic properties, particularly in the mouse model of hepatitis B compared to CS. This suggests that the secretome obtained by stimulating ASCs with disease-causing agents may have a more prominent therapeutic effect on the specific disease than the naïve secretome.

\section{Introduction}

Mesenchymal stem cells (MSCs) generally have antiinflammatory, tissue repairing and immunomodulatory properties (1). The therapeutic effect of MSCs is known to be mediated by their secretome (2-6). Secretome refers to the secretary molecules, cytokines, chemokines and growth factors that are released by MSCs and in broader terms, includes extracellular vesicles (EVs), such as exosomes, microvesicles and apoptotic bodies (3,5,7). Despite decades of research on stem cells, the paucity of clinical application is due to several limitations of stem cell research, including limited in vivo cell survival, senescence-related genetic instability, and potential of malignant transformation $(8,9)$. In this respect, utilizing the secretome instead of stem cells themselves is expected to be one of the prominent strategies which may be used to overcome the limitations of stem cell therapy $(8,10,11)$.

Adjusting the culture conditions of MSCs can lead to a significant difference in the composition of the secretome $(7,12)$. This means that a greater therapeutic potential could be obtained from the secretome with adjusted culture conditions than from the naïve secretome. There are mainly 2 methods use for adjusting the culture conditions of MSCs: The adjustment of the physico-chemical conditions and genetic engineering. As a method of improving the therapeutic potentials of MSCs by adjusting the physico-chemical conditions, the authors have previously attempted hypoxic and lipopolysaccharide pre-conditioning (13-15). In addition, several genes have been utilized to reinforce the therapeutic potentials of the secretome, including Akt (16), gene for heat shock protein 20 (17) and nuclear factor erythroid 2-related factor 2 (18). Overall, these methods attempted thus far belong to the non-specific stimulation of MSCs to generate the secretome. Furthermore, the authors attempted to identify a method which may be used to stimulate MSCs to generate a disease-specifical secretome. 
In the present study, it was hypothesized that pre-sensitization of MSCs with disease-causing agents could harness MSCs to release the secretome containing therapeutic materials specialized for the disease. Herein, the induced secretome (isecretome) was defined as the secretome released from MSCs that had been stimulated by disease-causing materials for the treatment of the specific disease. In a previous study, it was demonstrated that the thioacetamide (TAA; a hepatotoxin)-induced secretome was superior to the naïve secretome in restoring hepatic function, while minimizing inflammatory processes in mice with thioacetamide-induced hepatic failure (19). In the present study, the authors aimed to construct the isecretome using hepatitis B virus (HBV) to further generalize the hypothesis. If the $\mathrm{HBX}$-induced secretome (HBx-isecretrome) is shown to have a greater therapeutic efficacy than the control secretome in the model of hepatitis $\mathrm{B}$, it could increase the possibility of generating the disease-specific secretome.

\section{Materials and methods}

Chemicals, cells and cell culture. TAA was obtained from Sigma-Aldrich; Merck KGaA. The AML12 mouse hepatocyte cell line was obtained from the American Type Culture Collection (ATCC). AML12 cells were maintained in Dulbecco's modified Eagle's medium/Ham's F-12; DMEM/F12 (Thermo Fisher Scientific, Inc.). The medium was supplemented with 10\% FBS (fetal bovine serum; Gibco; Thermo Fisher Scientific, Inc.), $1 \%$ antibiotics (Thermo Fisher Scientific, Inc.), 1X ITS supplement (Insulin-Transferrin-Selenium-G supplement; Invitrogen; Thermo Fisher Scientific, Inc.) and $40 \mathrm{ng} / \mathrm{ml}$ dexamethasone (Sigma-Aldrich; Merck KGaA) at $37^{\circ} \mathrm{C}$. Human adipose-derived stem cells (ASCs) were kindly donated by Hurim BioCell Co. (Seoul). ASCs were cultured in DMEM/lowGlucose (Gibco; Thermo Fisher Scientific, Inc.) supplemented with antibiotics (Penicillin-streptomycin; Gibco; Thermo Fisher Scientific, Inc.) at $37^{\circ} \mathrm{C}$.

Preparation of secretomes released from ASCs. ASCs that reached $70-80 \%$ confluency were re-fed with serum-free DMEM low-glucose medium (Thermo Fisher Scientific, Inc.) at $37^{\circ} \mathrm{C}$ under $5 \% \mathrm{CO}_{2}$. Thereafter, the ASCs were cultured under 2 different conditions as follows: One subset was directly transfected with $4 \mu \mathrm{g}$ pcDNA-HBx for $24 \mathrm{~h}$ and the other subset was indirectly treated with culture medium from AML12 cells that had been transfected with $4 \mu \mathrm{g}$ pcDNA-HBx for $24 \mathrm{~h}$. In the process of transfection, pcDNA3.1 (Thermo Fisher Scientific, Inc.) we used as the plasmid backbone and Lipofectamine 2000 (Invitrogen; Thermo Fisher Scientific, Inc.) was used as a transfection reagent. In more detail, $24 \mathrm{~h}$ following the transfection of pcDNA-HBx into the ASCs, the transfected ASCs were incubated in serum-free DMEM low-glucose medium at $37^{\circ} \mathrm{C}$ for $24 \mathrm{~h}$. The 25 -fold-concentrated medium obtained from the culture medium of the transfected ASCs was termed as the direct pcDNA-HBx secretome. Subsequently, $24 \mathrm{~h}$ following the transfection of pcDNA-HBx into AML12 cells, the transfected AML12 cells were incubated in serum-free DMEM/F12 medium for $24 \mathrm{~h}$. The 25 -fold concentration of the culture medium obtained from the transfected AML12 cells was then obtained. Subsequently, the ASCs were treated with the 25-fold concentrated medium in serum-free DMEM low-glucose medium at a ratio of 1:100 for $24 \mathrm{~h}$. The culture medium of these ASCs was termed as the indirect pcDNA-HBx secretome. Finally, the control secretome (CS) refers to a 25 -fold concentration of the conditioned medium that was obtained following incubation of the ASCs for $24 \mathrm{~h}$. The concentration of the culture medium was accomplished using ultrafiltration units (Amicon Ultra-PL 3; EMD Millipore) with a 3-kDa cut-off.

Preparation of toxin-conditioned medium. The AML12 cells was treated with $0.1 \mathrm{mM}$ TAA for $24 \mathrm{~h}$. Conditioned medium from the TAA-treated AML12 cells were collected, and were concentrated approximately 25 -fold using ultra filtration units with a 3-kDa molecular weight cutoff. After culturing the ASCs that reached 70-80\% confluency, the cells were re-fed with serum-free DMEM low-glucose medium. The ASCs was treated with conditioned medium $(1: 1,000)$ for $24 \mathrm{~h}$. Toxin-conditioned medium from the CM-treated ASC cells was collected, and was concentrated approximately 25-fold using ultra filtration units with a $3-\mathrm{kDa}$ molecular weight cut-off. The toxin-conditioned medium was termed as the TAA-isecretome and stored at $-80^{\circ} \mathrm{C}$ until its use in the experiments.

Reverse transcription-quantitative PCR (RT-qPCR). Total cell RNA was extracted using FavorPrep TRI-RNA reagent (Favorgen) according to the manufacturer's instructions. Reverse transcription was performed with $1 \mu \mathrm{g}$ RNA using ReverTra Ace ${ }^{\circledR}$ qPCR RT Master mix (Toyobo Life Science) according to the manufacturer's instructions. The primers used for SYBR-Green quantitative PCR were as follows: Vascular endothelial growth factor (VEGF) sense, 5'-TGTACCTCC ACCATGCCAAGT-3' and antisense, 5'-CGCTGGTAGACA TCCATGAA-3'; hepatocyte growth factor (HGF) sense, 5'-TGCTGTCCTGGATGATTTTG-3' and antisense, 5'-AGT GTAGCCCCAGCCATAAA-3'; HBx sense, 5'-CCTCTACCG TCCCCTTCTTC-3' and antisense, 5'-GTCGGTCGTTGA CATTGTTG-3'; and GAPDH sense (forward), 5'-GCACCG TCAAGGCTGAGAAC-3' and antisense (reverse), 5'-TGG TGAAGACGCCAGTGGA-3'. The PCR parameters were as follows: An initial denaturation step (at $95^{\circ} \mathrm{C}$ for $10 \mathrm{sec}$ ); amplification and quantification step (40 cycles: At $95^{\circ} \mathrm{C}$ for $15 \mathrm{sec}$ and then $60^{\circ} \mathrm{C}$ for $\left.60 \mathrm{sec}\right)$; and final elongation step (1 cycle: At $95^{\circ} \mathrm{C}$ for $15 \mathrm{sec}, 60^{\circ} \mathrm{C}$ for $60 \mathrm{sec}$ and then $95^{\circ} \mathrm{C}$ for $15 \mathrm{sec}$ ). The reaction was performed using a Step one plus Real-Time PCR system (Life Technologies; Thermo Fisher Scientific, Inc.). Following normalization to the GAPDH gene, the expression levels for each target gene were calculated using the $2^{-\Delta \Delta \mathrm{Cq}}$ method (20).

Western blot analysis. Mouse liver specimens were lysed using the EzRIPA Lysis kit (ATTO Corporation), and quantified using Bradford reagent (Bio-Rad Laboratories, Inc.). Cells and liver tissue lysates were separated by SDS-polyacrylamide gel on $10 \%$ gels and electrotransferred onto a nitrocellulose membrane (Bio-Rad Laboratories, Inc.). The membrane was blocked with $1 \mathrm{X}$ blocking solution (TransLab Biosciences) for $1 \mathrm{~h}$ at room temperature. Proteins were visualized by western blot analysis by incubation with the following primary antibodies 
(1:1,000 dilution) at $4^{\circ} \mathrm{C}$ overnight and then with horseradish peroxidase (HRP)-conjugated secondary antibodies (1:2,000 dilution) for $1 \mathrm{~h}$ at $25^{\circ} \mathrm{C}$. Primary antibodies against Bcl-2-like protein 11 (Bim, cat no. 2933), HGF, myeloid cell leukemia 1 (Mcl-1, cat no. 5453), poly(ADP-ribose) polymerase (PARP, cat no. \#9542), proliferating cell nuclear antigen (PCNA, cat no. 2586), phosphorylated signal transducer and activator of transcription 3 (p-STAT3, cat no. 9131) and cleaved caspase 3 (c-caspase 3, cat no. 9664) were obtained from Cell Signaling Technology, Inc. VEGF (cat no. ab46154) was obtained from Abcam. $\beta$-actin (cat no. sc58673) was obtained from Santa Cruz Biotechnology, Inc. HRP-conjugated secondary antibody (cat no. ADI-SAB-100) was obtained from Enzo Life Sciences. Specific immune complexes were detected using the Western Blotting Plus Chemiluminescence Reagent (EMD Millipore). Relative densities of individual markers were quantified using Image Lab software V3.0 (Bio-Rad Laboratories, Inc.).

Animal experiments. BALB/c mice, weighing 25-30 g ( $\mathrm{n}=52$; 8 weeks old, male; Damool Science) were used in the present study. The Ethics Committee at Daejeon St. Mary's Hospital, the Catholic University of Korea, approved the animal experiments for the research (IRB no. CMCDJ-AP-2016-001). The in vivo model of chronic hepatitis $\mathrm{B}$ was generated by an intravenous injection of pcDNA-HBx (4 $\mu \mathrm{g}$ pcDNA-HBx and in vivo-jetPEI ${ }^{\circledR}$ Transfection mix, once a week for 6 weeks) into the mice, as previously described $(21,22)$. Subsequently, the experimental mice received 4 intravenous injections (twice a week for 2 weeks through the tail vein) of secretome (100 $\mu \mathrm{l}$ for each injection). Specifically, the control mice were injected with normal saline (control group; $\mathrm{n}=5$ ), the control secretome (CS; $\mathrm{n}=5), \mathrm{HBx}-\mathrm{DS}(\mathrm{n}=5)$ and HBx-IS ( $=5)$, and the mice with hepatitis B were injected with normal saline (control group; $n=8$ ), CS $(n=8), H B x-D S(n=8)$ and HBx-IS $(n=8)$ (Fig. 1A). Herein, $\mathrm{CS}$ refers to a 25 -fold concentration of the conditioned medium that was obtained following incubation of the ASCs at $37^{\circ} \mathrm{C}$ for $24 \mathrm{~h}$. Subsequently, the mouse model of TAA-induced hepatic failure was generated using TAA (each amount, $300 \mathrm{mg} / \mathrm{kg}$ ) for 2 consequent days administered intraperitoneally. From the first day of the TAA administration, the mice received 2 intravenous injections (100 $\mu \mathrm{l}$ for each injection) of CS ( $\mathrm{n}=5)$, TAA-isecretome $(n=5)$ and HBx-IS $(n=5)$ for 2 consequent days, via the tail vein. On the following day of the final injection, the mice were euthanized and the liver specimens were examined.

Serological test and ELISA. Blood samples $(0.2 \mathrm{ml}$ per one time) obtained from each mouse from the tail vein on the day of euthanization, were centrifuged at $4^{\circ} \mathrm{C}$ for $10 \mathrm{~min}$ at $10,000 \mathrm{x} \mathrm{g}$ and the sera were collected. The concentrations of markers of liver injury, such as aspartate transaminase (AST) and alanine transaminase (ALT) were measured using an IDEXX VetTest Chemistry Analyzer (IDEXX Laboratories, Inc.). In addition, the serum concentrations of mouse interleukin (IL)- 6 and tumor necrosis factor- $\alpha$ (TNF- $\alpha$ ) were measured using a sandwich enzyme-linked immunosorbent (ELISA) assay (Abcam, Inc.) according to the manufacturer's instructions.

Hematoxylin and eosin (H\&E) staining, immunohistochemistry and immunofluorescence. For analysis, formalin-fixed paraffin-embedded liver tissue sections ( $<3 \mathrm{~mm}$ in thickness) were deparaffinized, rehydrated in an ethanol series and subjected to epitope retrieval using standard procedures. H\&E staining was performed by staining the specimens with Harris's hematoxylin (YD Dignostics) for $7 \mathrm{~min}$, rinsing with tap water for $1 \mathrm{~min}$, and incubation with Eosin Y solution (Duksan) for $150 \mathrm{sec}$ at $25^{\circ} \mathrm{C}$. The sections were then dehydrated with $80 \%$ and then wtih $100 \%$ EtOH, dipped in Xylene (Duksan) for $3 \mathrm{~min}$ and mounted in a permount mounting medium (cat. no. \#SP15-100; Thermo Fisher Scientific, Inc.). The specimens were examined under a panoramic distal slide scanner system (3D HISTECH). Immunohistochemical analysis were visualized by incubation with the following primary antibodies at $4^{\circ} \mathrm{C}$ overnight and then with peroxidase-conjugated secondary antibodies (cat nos. PK-6101 and PK-6102; Vector Laboratories, Inc.) for $1 \mathrm{~h}$ at $25^{\circ} \mathrm{C}$. Antibodies against VEGF (1:200; Abcam, cat no. ab1316), B-cell leukemia-extra large (Bcl-xL) (1:100; Abcam, ab98143) and $\gamma$-glutamyl transpeptidase ( $\gamma$-GTP; Santa Cruz Biotechnology, Inc., 1:200; cat no. sc100746) were used for immunochemical staining. The samples were then examined under a panoramic distal slide scanner system (3D HISTECH). immunofluorescence analysis were visualized by incubation with the following primary antibodies at $4{ }^{\circ} \mathrm{C}$ overnight and then with fluorescent-secondary antibodies (1:500; Invitrogen; Thermo Fisher Scientific, Inc., cat no. A11005 and A11008) for $1 \mathrm{~h}$ at $25^{\circ} \mathrm{C}$. Antibodies against F4/80 (1:100; Santa Cruz Biotechnology, Inc., cat no. sc37709) and CD68 (1:500; Abcam, cat no. \#125212) were used for immunofluorescence staining. The samples were then examined under a fluorescence imaging system (EVOS M5000: Thermo Fisher Scientific, Inc.) to analyze the expression of F4/80 and CD68. Percentages of immunofluorescent areas were measured using NIH Image $\mathbf{J}$ and expressed as relative values to those in control mice.

Statistical analysis. All data were analyzed using SPSS 11.0 software (SPSS Inc.), and are presented as the means \pm standard deviation (SD). Statistical comparison among groups was determined using the Kruskal-Wallis test followed by the Mann-Whitney test with Bonferroni correction as the post hoc analysis. A P-value $<0.05$ was considered to indicate a statistically significant difference.

\section{Results}

Effects of $H B x$-isecretome on the expression of HGF and $V E G F$ mRNA in the liver. The present study aimed to determine whether the secretome obtained by stimulating ASCs with specific disease-causing agents (pathogens) was more effective in improving the specific disease than the control secretome. In this experiment, the pathogen and the specific disease were set to HBx and hepatitis B, respectively. The present study used 2 methods to obtain the HBx-isecretome. The first method involved the collection of the secretome released from $\mathrm{HBx}$-overexpressing ASCs that had been produced by transfecting ASCs with pcDNA-HBx for $24 \mathrm{~h}$. The second method involved the collection of secretary materials following the stimulation of ASCs with 100-fold diluted culture medium of AML12 cells that had been transfected with pcDNA-HBx for $24 \mathrm{~h}$ (Fig. 1B). These were termed as the direct $\mathrm{HBx}$-isecretome 
Five injections of pcDNA-HBx (once a week for 6 weeks)
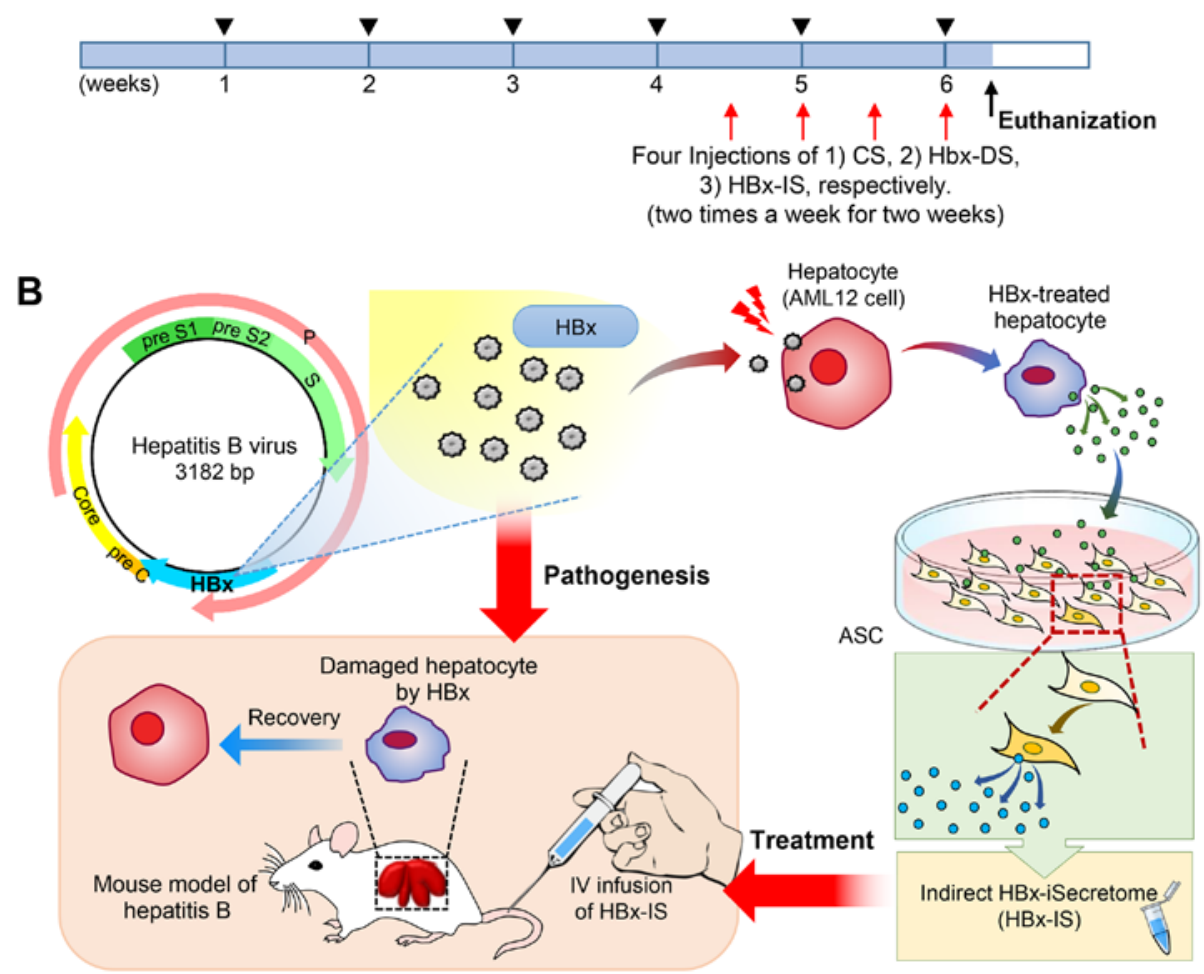

Figure 1. Overview of the study design. (A) Schedule of modeling and intervention. (B) Study design. The mouse model of hepatitis B was generated by injecting pcDNA-HBx via the tail vein weekly for 6 weeks. Intervention was achieved by injecting CS, HBx-DS, and HBx-IS via tail vein four times (twice a week for 2 weeks), respectively. HBx, hepatitis B virus protein X; CS, control secretome; HBx-IS, indirect HBx-induced secretome; HBx-DS, direct HBx-induced secretome; iSecretome, induced secretome.

(HBx-DS) and indirect $\mathrm{HBx}$-isecretome (HBx-IS), respectively. The successful transfection of HBx was identified by confirming the upregulation of HBx through RT-qPCR. Specifically, in vitro transfection was validated by demonstrating the higher HBx-mRNA expression in the transfected AML12 cells and ASCs, respectively (both P $<0.05$; Fig. 2A). Subsequently, in vivo transfection was validated by demonstrating the higher HBx-mRNA expression in the liver tissues of the mice that had been injected with pcDNA-HBx via the tail vein $(\mathrm{P}<0.05$; Fig. $2 \mathrm{~B})$. An animal model of hepatitis $\mathrm{B}$ was then generated by injecting $\mathrm{HBx}$ into mice, and the mice were subsequently intravenously administered CS, HBx-DS and HBx-IS. On the following day of the final injection, the mice were euthanized and the specimens were investigated. H\&E staining of each treatment revealed that HBx-IS infusion resulted in a higher restoration of the hepatic tissue than HBx-DS infusion (Fig. 2C). RT-qPCR was performed to compare the mRNA expression levels of HGF and VEGF in the mouse liver specimens (Fig. 2D). The HBx-DS injection did not increase the mRNA expression of HGF and VEGF compared to the CS injection in the mice with hepatitis B. However, the HBx-IS injection to the mice with hepatitis B significantly increased the mRNA expression of HGF and VEGF compared to the CS injection $(\mathrm{P}<0.05)$.

Effects of the HBx-isecretome on the serum levels of liver enzymes and pro-inflammatory cytokines. Sera from mice were collected at the day of euthanization and the serum levels of liver enzymes and pro-inflammatory cytokines were compared. The mice with hepatitis B exhibited elevated levels of AST, which were significantly decreased following the individual secretome treatments (CS, HBx-DS and HBx-IS) $(\mathrm{P}<0.05$; Fig. 3A). The serum levels of ALT were not significantly increased in the mice with hepatitis $\mathrm{B}$, and were significantly decreased following the individual secretome treatments $(\mathrm{P}<0.05)$. Subsequently, the serum levels of IL-6 and TNF- $\alpha$ were compared in each group (Fig. 3B). In the mice with hepatitis $\mathrm{B}$, the HBx-DS injection did not significantly reduce the serum levels of IL- 6 and TNF- $\alpha$ compared to the CS injection. However, the HBx-IS injection significantly decreased the serum levels of IL- 6 and TNF- $\alpha$ compared to the CS injection $(\mathrm{P}<0.05)$.

Effects of the HBx-isecretome on the expression of proteins reflecting liver regeneration. Western blot analysis was performed to determine the expression of liver regeneration-related proteins in the liver specimens following each treatment. The liver regeneration-related proteins, included p-STAT3/t-STAT3, VEGF, HGF and PCNA. In the mice with hepatitis B, HBx-DS did not significantly increase the expression levels of these liver regeneration-related proteins compared with the CS (Fig. 4A). By contrast, HBx-IS significantly increased the expression levels of p-STAT3/t-STAT3 and PCNA compared with the CS ( $\mathrm{P}<0.05$; Fig. 4B).

Effects of the HBx-isecretome on the expression of apoptosis-related proteins. Western blot analysis was further performed to determine the expression levels of 
A

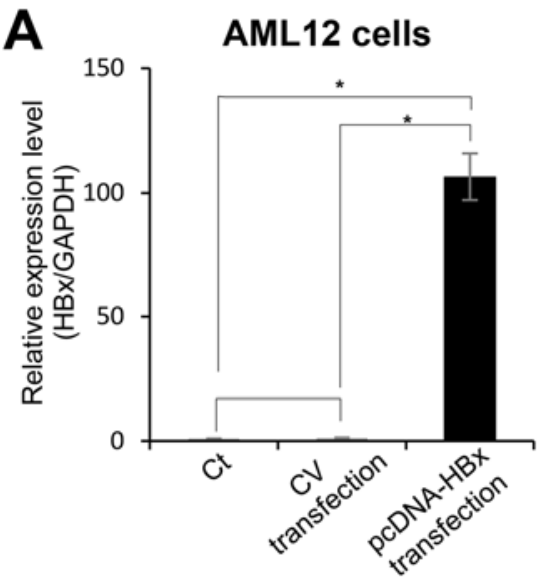

C

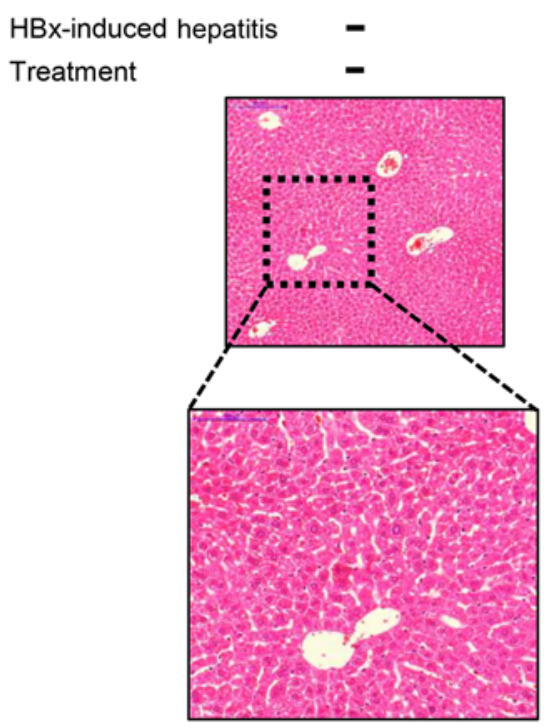

D

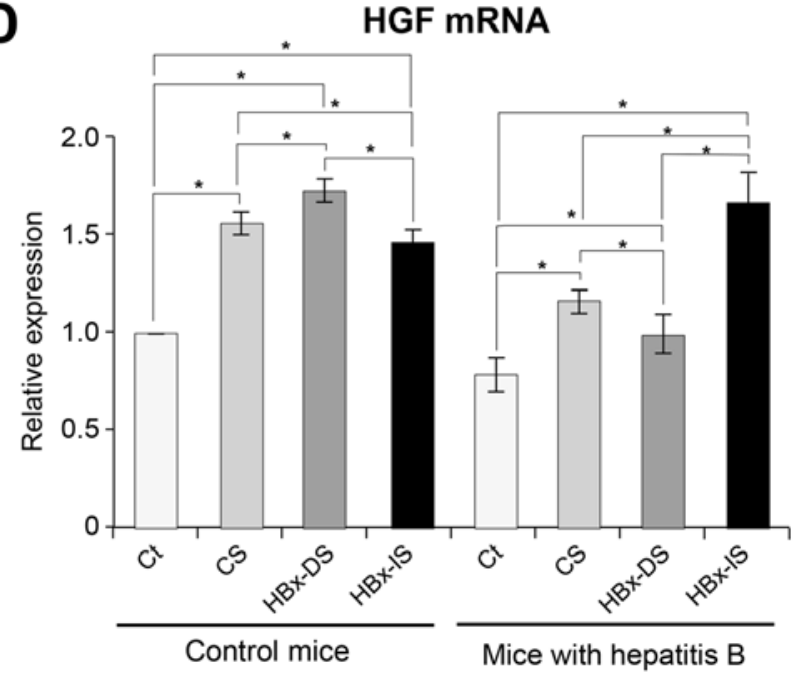

ASCs

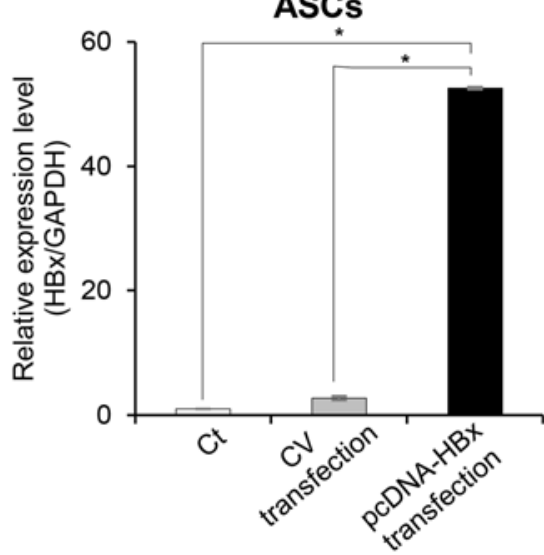

B
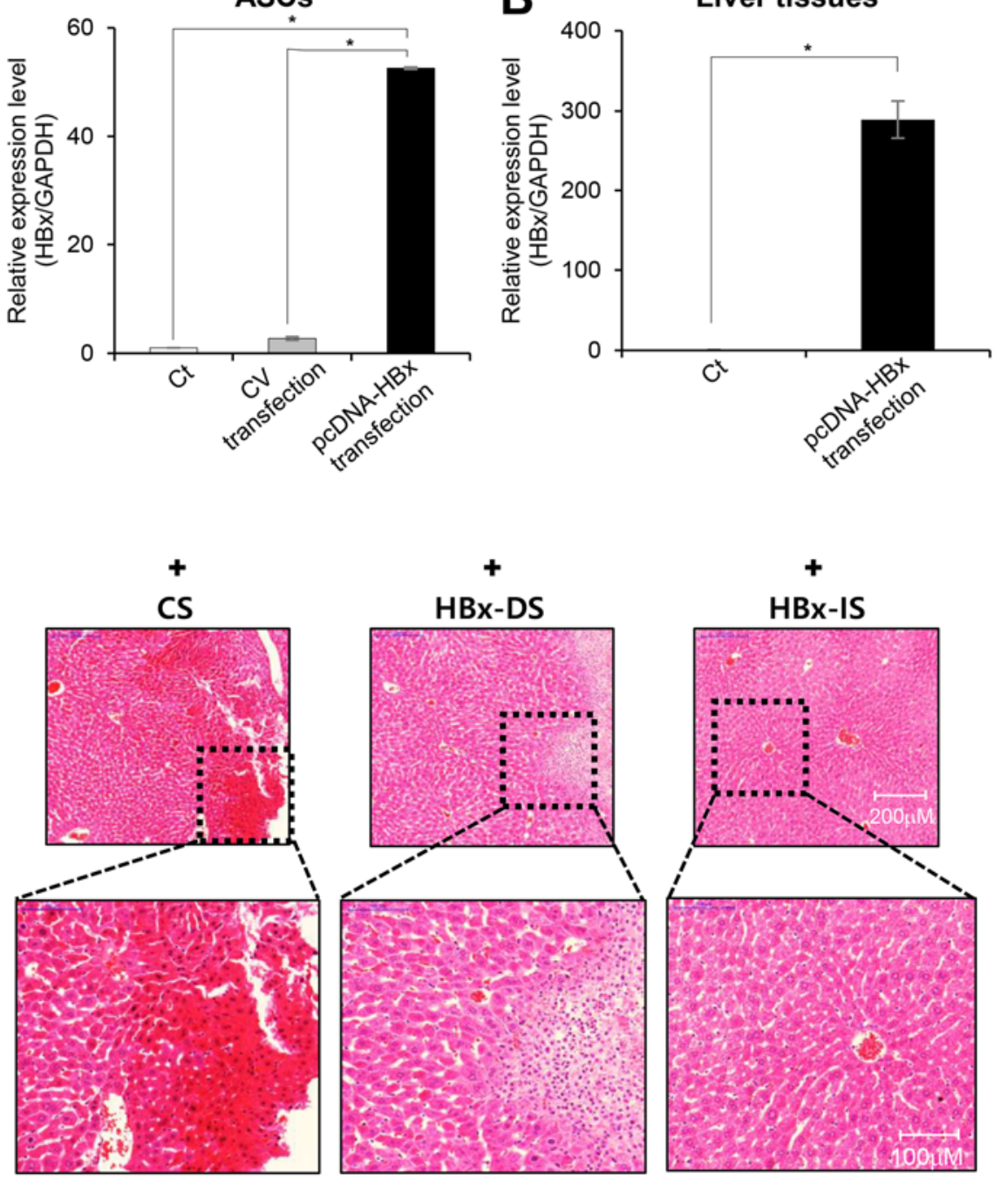

Liver tissues

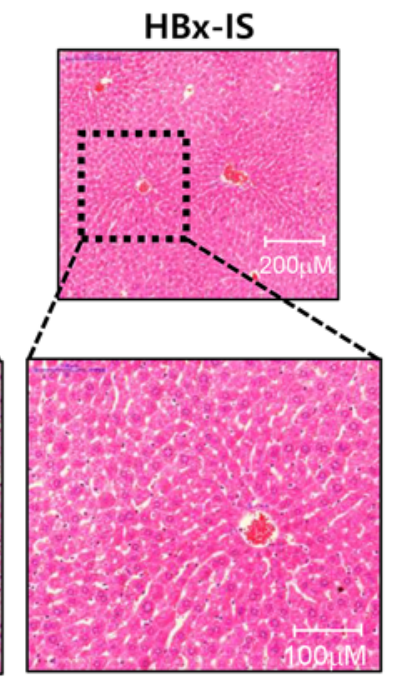

VEGF mRNA

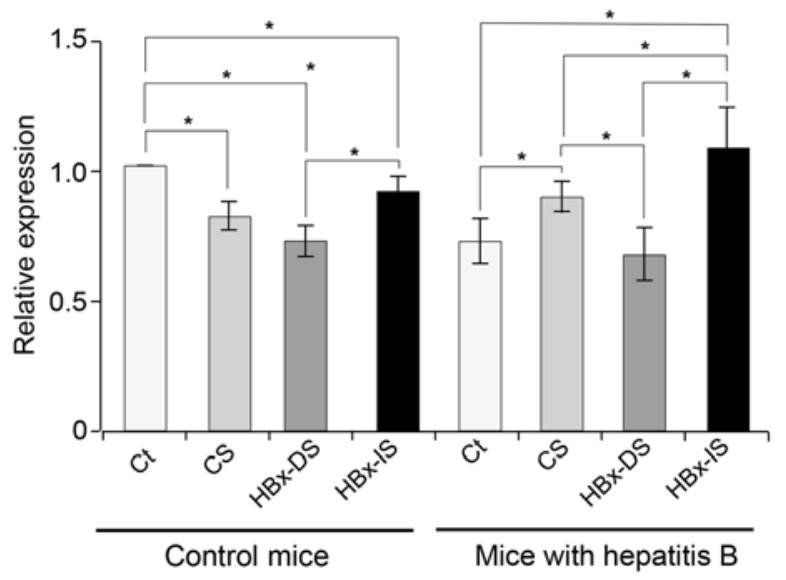

Figure 2. Validation of HBx transfection and the effects of the HBx-isecretome on the expression of mRNAs reflecting liver regeneration. (A) In vitro validation of HBx transfection. Results of RT-qPCR showing the higher HBx-mRNA expression in the transfected AML12 cells and ASCs, respectively (both P<0.05). (B) In vivo validation of HBx transfection. Results of RT-qPCR showing the higher HBx-mRNA expression in the liver tissues of the mice that had been injected with pcDNA-HBx via the tail vein $(\mathrm{P}<0.05)$. (C) Representative histological comparison of each treatment. CS, HBx-DS and HBx-IS, respectively, were intravenously administered to the mice with $\mathrm{HBx}$-induced hepatitis. H\&E staining showing that the HBx-IS infusion resulted in a greater restoration of the hepatic tissue than the HBx-DS infusion. (D) Results of RT-qPCR showing the mRNA expression of HGF and VEGF following individual treatments. HBx-DS injection did not increase the expression of HGF and VEGF mRNA compared to the CS injection in the mice with hepatitis B. However, the HBx-IS injection to the mice with hepatitis B significantly increased the expression of HGF and VEGF mRNA compared to the CS injection. Values are presented as the means \pm standard deviation of 3 independent experiments. ${ }^{*} \mathrm{P}<0.05$. ASCs, adipose-derived stem cells; HBx, hepatitis B virus protein $\mathrm{X}$; CS, control secretome; CV, control vector; HBx-IS, indirect HBx-induced secretome; HBx-DS, direct HBx-induced secretome; HGF, hepatocyte growth factor; VEGF, vascular endothelial growth factor. 

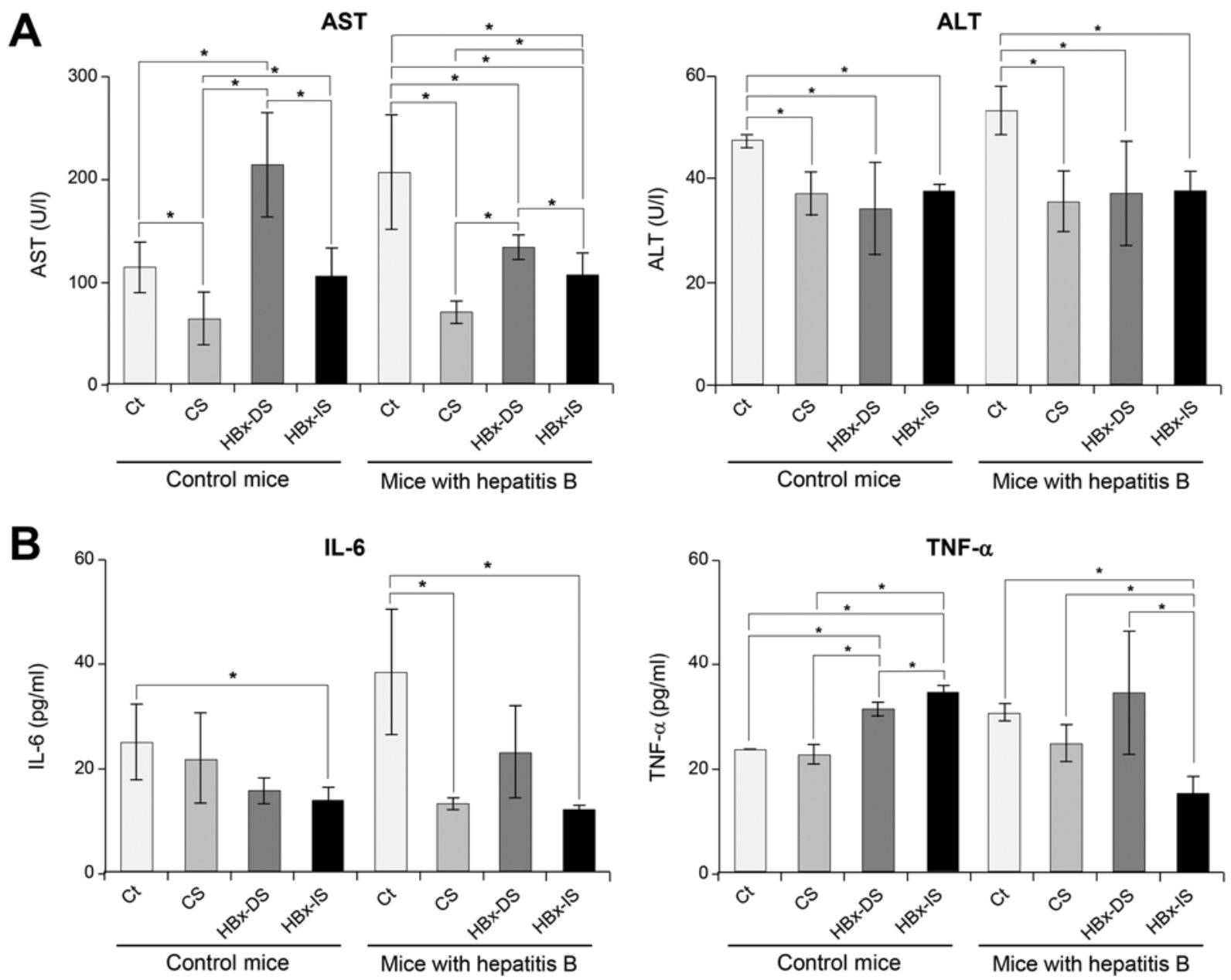

Figure 3. Effects of the HBx-isecretome on the serum levels of liver enzymes and pro-inflammatory cytokines. (A) Comparison of serum levels of liver enzymes following each treatment. (B) Results of EILSA showing serum levels of pro-inflammatory cytokines following each treatment. Values are presented as the means \pm standard deviation of 3 independent experiments. ${ }^{~} \mathrm{P}<0.05$. ALT, alanine transaminase; AST, aspartate transaminase; HBx, hepatitis B virus protein X; CS, control secretome; HBx-IS, indirect HBx-induced secretome; HBx-DS, direct HBx-induced secretome; TNF- $\alpha$, tumor necrosis factor- $\alpha$.

apoptosis-related proteins in the liver specimens following each treatment. Pro-apoptotic markers included PARP, c-caspase 3 and BIM, and the examined anti-apoptotic marker, was Mcl-1. In the mice with hepatitis B, HBx-DS significantly increased the expression of certain pro-apoptotic markers (c-caspase 3 and BIM) and significantly decreased the expression of Mcl-1 compared with the CS ( $\mathrm{P}<0.05$; Fig. 5A). By contrast, HBx-IS significantly decreased the expression of all the pro-apoptotic markers tested $(\mathrm{P}<0.05)$, and insignificantly increased Mcl-1 compared with the CS (Fig. 5B).

Immunohistochemistry of the liver following the administration of the HBx-isecretome. Immunostaining for VEGF (a liver regeneration-related protein), Bcl-xL (an anti-apoptotic protein) and $\gamma$-GTP (a marker for hepatitis) was performed using the liver specimens. The mouse model of hepatitis B exhibited a decreased expression of VEGF and Bcl-xL in the liver. However, the injection of CS and HBx-IS increased the expression of VEGF and Bcl-xL in the liver (Fig. 6A and B). When comparing the CS and HBx-IS, the expression of VEGF and Bcl-xL was significantly higher in the HBx-IS group than in the CS group $(\mathrm{P}<0.05)$. By contrast, the mouse model of hepatitis B exhibited an increased expression of $\gamma$-GTP in the liver. However, the injection of CS and HBx-IS decreased the expression of $\gamma$-GTP in the liver (Fig. 6C). When comparing CS and HBx-IS, the expression of $\gamma$-GTP was significantly lower in the HBx-IS group than in the CS group $(\mathrm{P}<0.05)$. Taken together, these results suggest that HBx-IS has a higher liver regenerative potential and a higher ability to inhibit cell apoptosis and hepatitis than CS.

Immunofluorescence of the liver following the administration of the HBx-isecretome. Immunostaining for pro-inflammatory markers, such as F4/80 and CD68, in the liver specimens was finally performed (Fig. 7A and B). The mouse model of hepatitis B exhibited an increased expression of these markers in the liver. However, the injection of CS and HBx-IS significantly decreased the expression levels of these markers in the liver $(\mathrm{P}<0.05)$. When comparing CS and HBx-IS, the expression of these markers was significantly lower in the HBx-IS group compared to the CS group $(\mathrm{P}<0.05)$, suggesting that the HBx-IS injection inhibited the inflammatory reactions in the liver more effectively than the CS injection.

Validation of the disease-specificity of the HBx-isecretome. To validate the disease-specific effectiveness of the 
A

HBx-DS

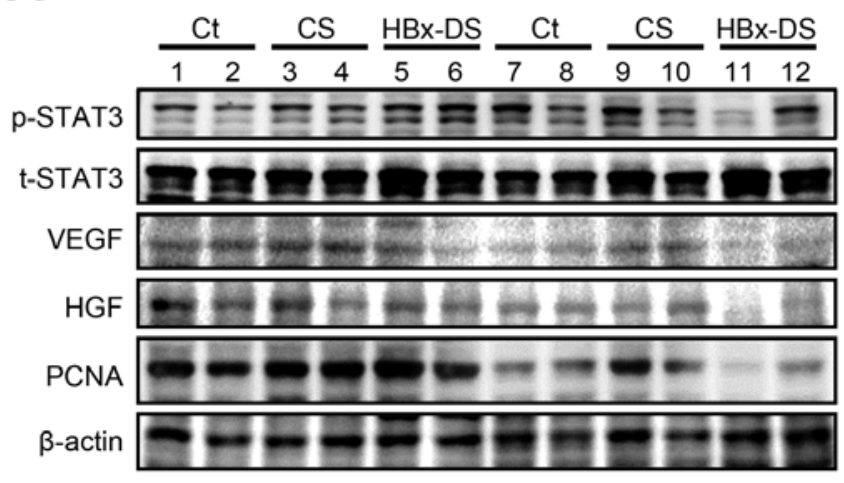

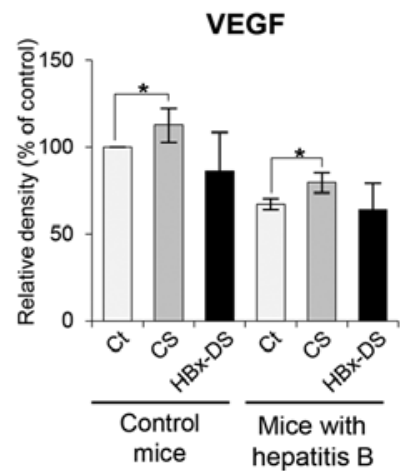

PCNA
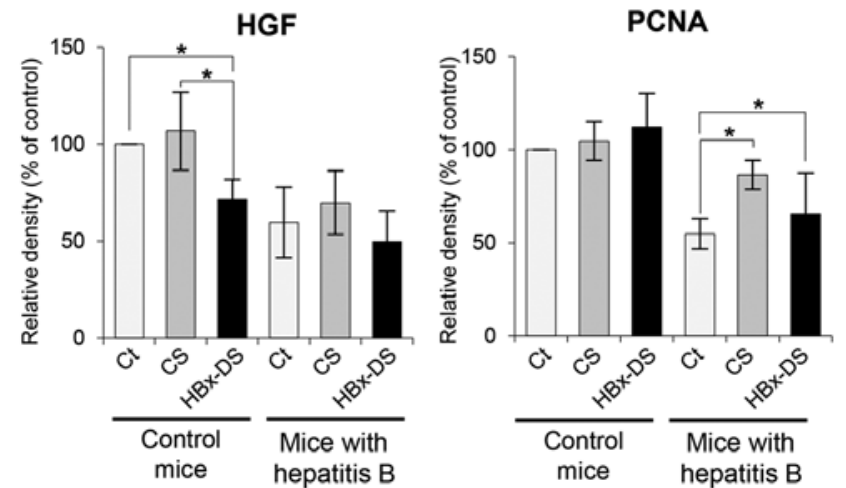

B

HBx-IS

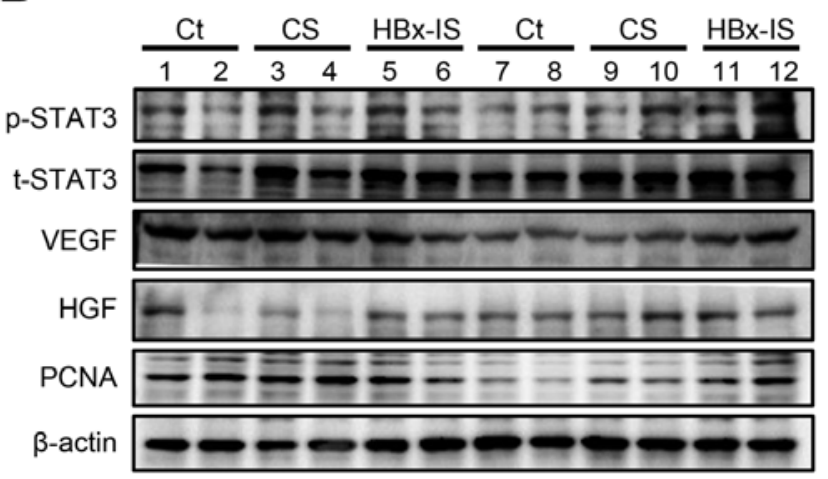

Figure 4. Effects of the HBx-isecretome on the expression of proteins reflecting liver regeneration. (A) Top panel illustrates the effects of HBx-DS on the expression of proteins reflecting liver regeneration. In the mice with hepatitis B, HBx-DS did not significantly increase the expression of these liver regeneration-related proteins compared with CS. Bottom panel illustrates relative densities of proteins reflecting liver regeneration in each group. (B) Top panel illustrates the effects of HBx-IS on the expression of proteins reflecting liver regeneration. HBx-IS significantly increased the expression of p-STAT3/t-STAT3 and PCNA compared with CS. Bottom panel illustrates relative densities of proteins reflecting liver regeneration in each group. Values are presented as the means \pm standard deviation of 3 independent experiments. "P<0.05. Relative densities of individual markers were quantified using Image Lab software and were then normalized to those of $\beta$-actin in each group. HBx, hepatitis B virus protein X; CS, control secretome; HBx-IS, indirect HBx-induced secretome; HBx-DS, direct HBx-induced secretome; HGF, hepatocyte growth factor; PCNA, proliferating cell nuclear antigen; STAT3, Signal transducer and activator of transcription 3; VEGF, vascular endothelial growth factor.

HBx-isecretome, the control secretome, TAA-isecretome and $\mathrm{HBx}$-isecretome, we intravenously infused into the mice in the model of TAA-induced hepatic failure. At 2 days after the first infusion, the mice were euthanized and the specimens were examined. Western blot analysis revealed that the TAA-isecretome infusion, rather than the $\mathrm{HBx}$-isecretome infusion, induced a higher expression of proliferation markers (HGF, VEGF and PCNA) (all P<0.05; Fig. 8). Taken together, whereas the HBx-isecretome exerted the optimal proliferative and anti-inflammatory effects against HBx-induced hepatic failure, its capacity for promoting the recovery of the liver appeared to be much inferior to the TAA-isecretome in mice with TAA-induced hepatic failure, suggestive of the potential for disease-specific treatment.

\section{Discussion}

The secretome released by ASCs has the potential to induce tissue regeneration and repair. The present study attempted to identify a method which may be used to enhance the 
A
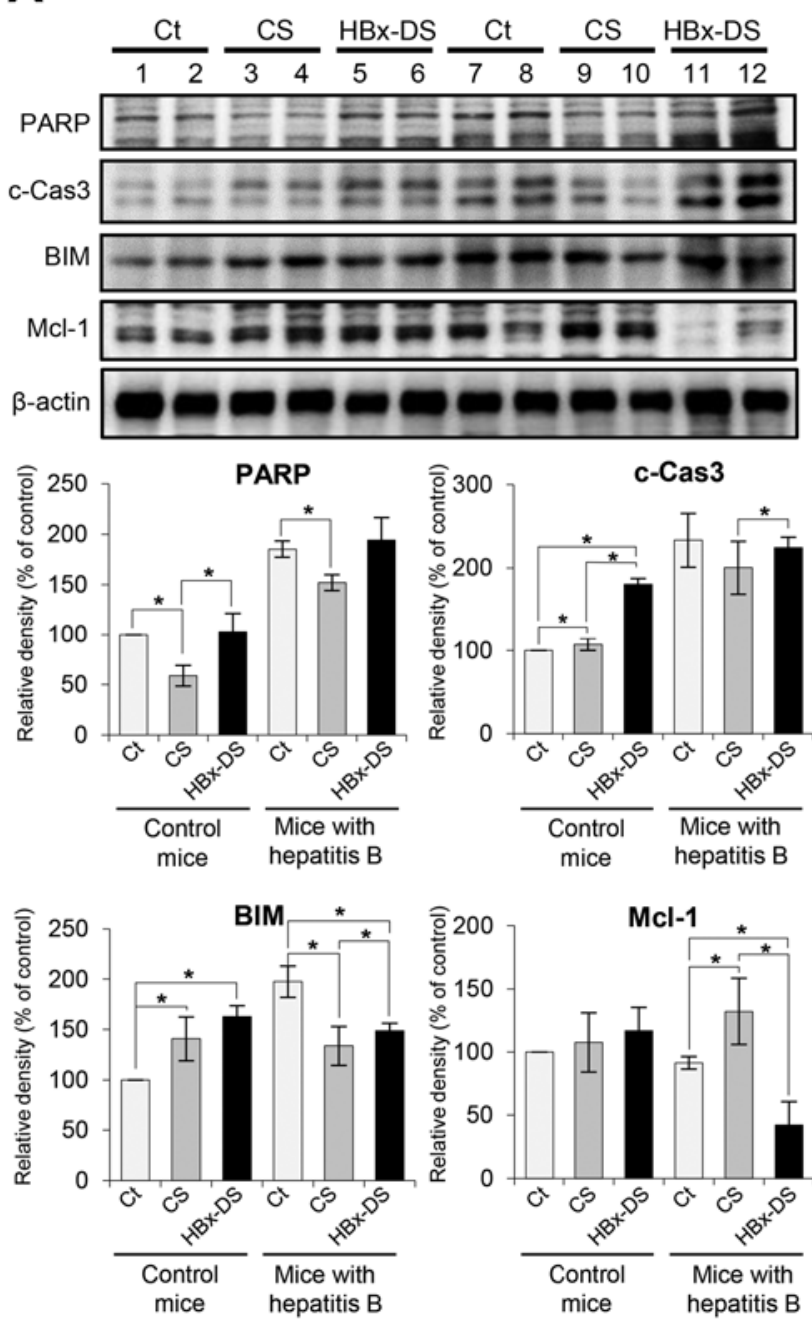

HBx-DS

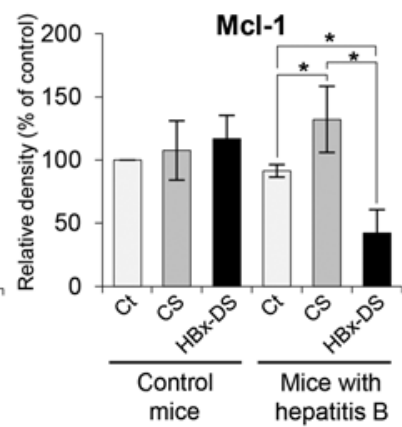

B
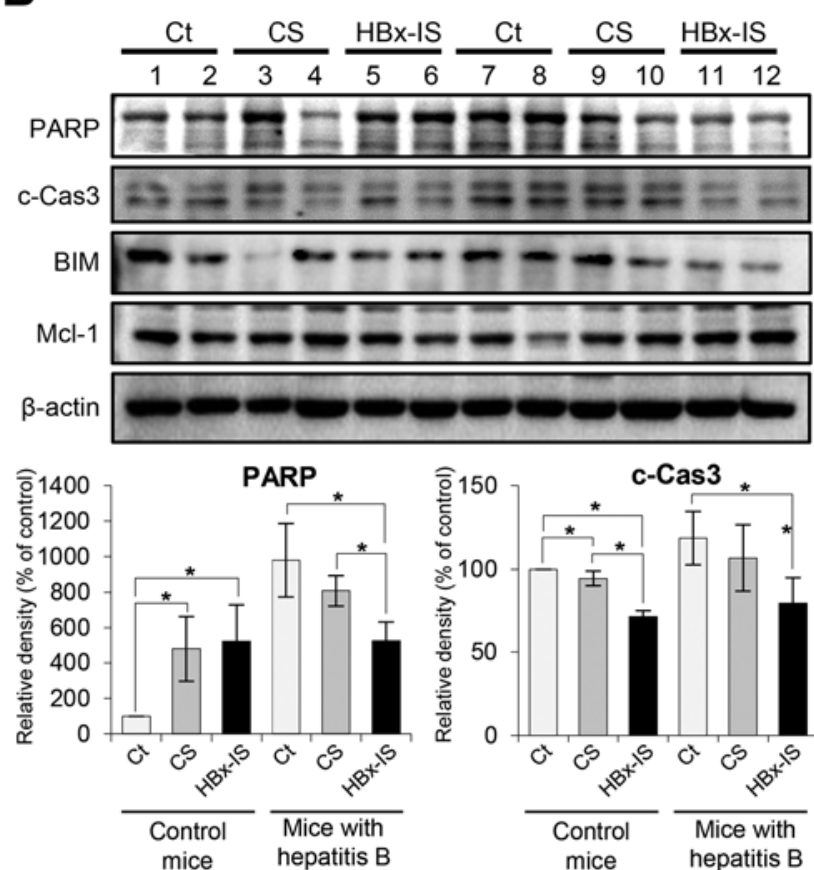
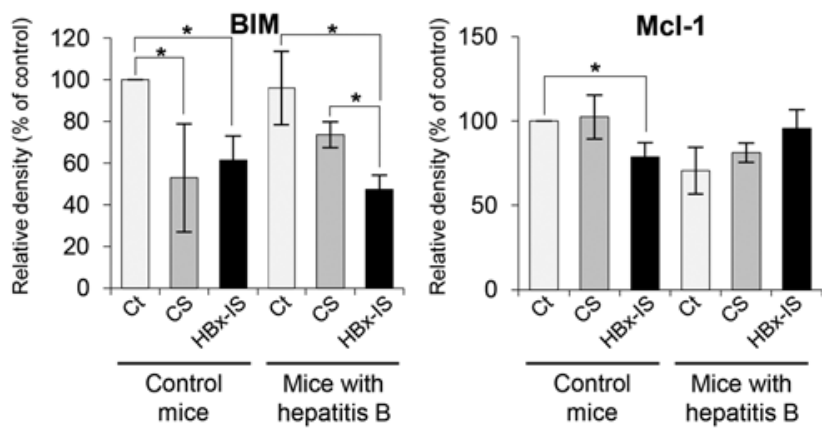

Figure 5. Effects of the HBx-isecretome on the expression of apoptosis-related proteins. (A) Top panel illustrates the effects of HBx-DS on the expression of apoptosis-related proteins. In the mice with hepatitis B, HBx-DS significantly increased the expression of certain pro-apoptotic markers (c-caspase 3 and BIM) and significantly decreased the expression of Mcl-1 compared with CS. Bottom panel illustrates relative densities of apoptosis-related proteins in each group. (B) Top panel illustrates the effects of HBx-IS on the expression of apoptosis-related proteins. HBx-IS significantly decreased the expression of all the pro-apoptotic markers tested $(\mathrm{P}<0.05)$, and insignificantly increased Mcl-1 compared with CS. Bottom panel illustrates relative densities of apoptosis-related proteins in each group. Values are presented as the means \pm standard deviation of 3 independent experiments. Relative densities of individual markers had been quantified using Image Lab software and were then normalized to those of $\beta$-actin in each group. ${ }^{*} \mathrm{P}<0.05$. BIM, Bcl-2-like protein 11 ; HBx, hepatitis B virus protein X; c-Cas3, cleaved caspase-3; CS, control secretome; HBx-IS, indirect HBx-induced secretome; HBx-DS, direct HBx-induced secretome; Mcl-1, myeloid cell leukemia 1; PARP, poly-ADP (adenosine diphosphate)-ribose polymerase.

disease-specific therapeutic effect of the secretome. Specifically, the therapeutic potential of the $\mathrm{HBx}$-isecretome in a mouse model of hepatitis $B$ was determined. The HBx-isecretome (HBx-IS) was obtained by collecting the secretary materials following the stimulation of ASCs with 100 -fold diluted culture medium of AML12 cells that had been transfected with pcDNA-HBx for $24 \mathrm{~h}$. Subsequently, HBx-IS was intravenously administered to the mice with hepatitis B. Compared with the CS injection, the HBX-IS injection more significantly reduced tje serum levels of IL-6 and TNF- $\alpha$ (pro-inflammatory cytokines). The HBx-IS injection led to the higher expression of liver regeneration-related proteins and to the lower expression of pro-inflammatory and pro-apoptotic proteins in mouse livers than the CS injection. Taken together, these results indicate that HBx-IS exhibits greater liver regenerative, anti-inflammatory and anti-apoptotic properties, particularly in the mouse model of hepatitis B than the CS. This suggests that secretomes obtained by stimulating ASCs with disease-causing agents could have a more promiment therapeutic effect in specific disease than naïve secretomes.

In general, cells have the property of protecting themselves when exposed to irritants or toxins. This property can be expressed by the release of secretomes in response to external stimuli (23-26). For example, when exposed to live toxins, primary hepatocytes are known to release large amounts of vital liver-specific proteins, including the enzymes carbamoyl phosphate synthetase $1, \mathrm{~S}$-adenosyl methionine synthetase 1 and catechol- $O$-methyltransferase (27). However, there is a marked difference in the amount and composition of the secretomes released from mature cells and MSCs against the same stimuli. Since MSCs have a stronger responsiveness and plasticity against external stimuli than mature cells, they generally produce larger amounts of secretome with greater 
A

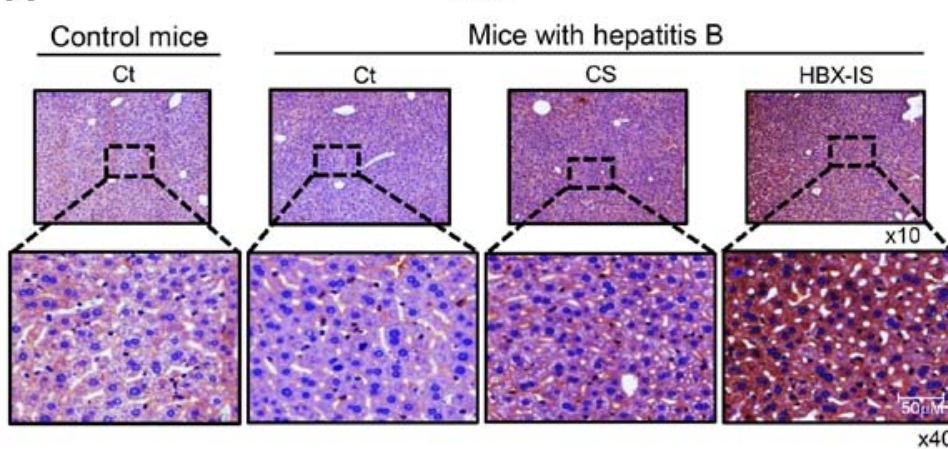

B

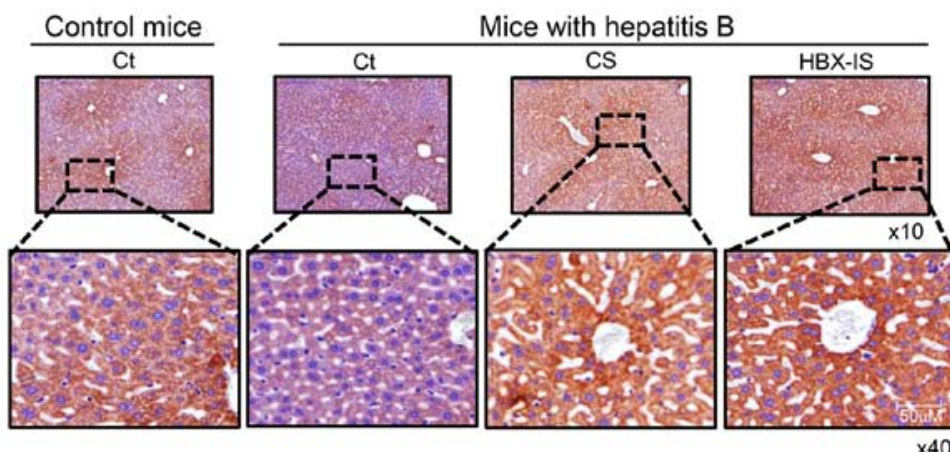

C

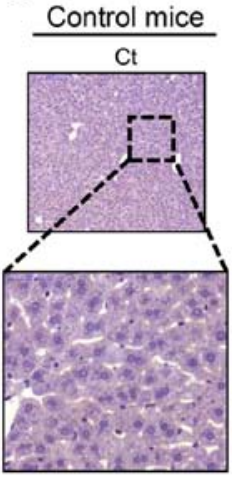

Bcl-xL

$\mathrm{x} 40$

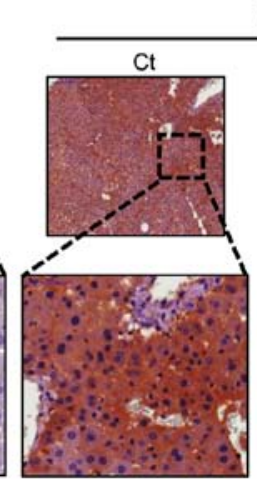

Mice with hepatitis B
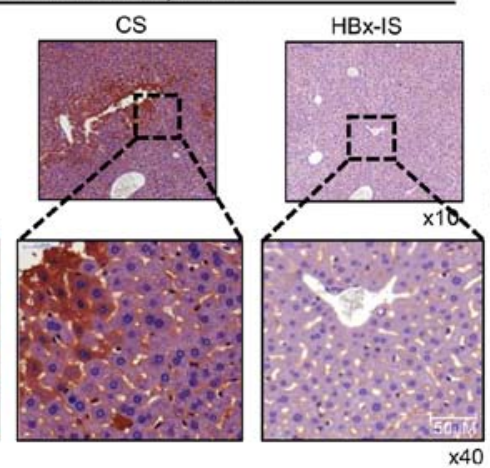

VEGF

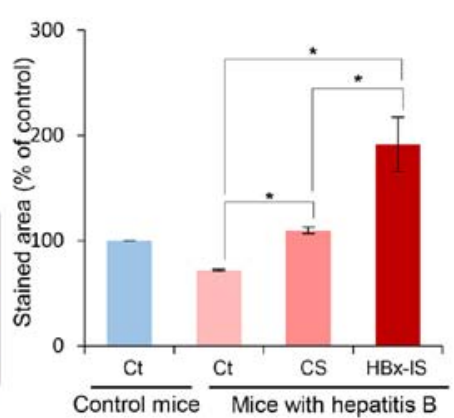

Bcl-xL

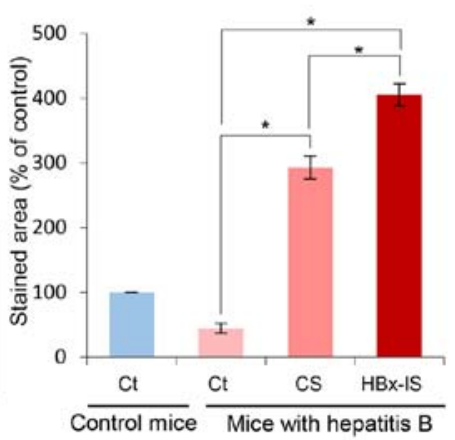

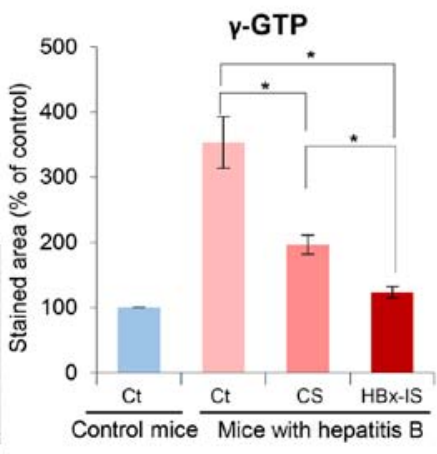

Figure 6. Immnohistochemical stains of the liver following administration of the HBx-isecretome. (A) Left panel illustrates VEGF immunohistochemistry of the liver following the administration of each treatment. Injection of CS and HBx-IS both significantly increased the expression of VEGF in the liver. In addition, the expression of VEGF was significantly higher in the HBx-IS group than in the CS group. Right panel presents the percentages of VEGF immunoreactive areas. (B) Left panel illustrates Bcl-xL immunohistochemistry of the liver following administration of each treatment. Injection of CS and HBx-IS both significantly increased the expression of Bcl-xL in the liver. In addition, the expression of Bcl-xL was significantly higher in the HBx-IS group than in the CS group. Right panel presents the percentages of Bcl-xL immunoreactive areas. (C) Left panel illustrates $\gamma$-GTP immunohistochemistry of the liver following administration of each treatment. Injection of CS and HBx-IS both significantly decreased the expression of $\gamma$-GTP in the liver. When comparing CS and HBx-IS, HBx-IS, the expression of $\gamma$-GTP was significantly lower in the HBx-IS group than in the CS group. Right panel presents percentages of $\gamma$-GTP immunoreactive areas. Values are presented as the means \pm standard deviation of 3 independent experiments. Percentages of immunoreactive areas were measured using NIH image $\mathrm{J}$ and expressed as relative values to those in normal livers. ${ }^{*} \mathrm{P}<0.05$. Bcl-xL, B-cell leukemia-extra large; HBx, hepatitis B virus protein X; CS, control secretome; $\gamma$-GTP, $\gamma$-glutamyltranspeptidase; HBx-IS, indirect HBx-induced secretome; HBx-DS, direct HBx-induced secretome; VEGF, vascular endothelial growth factor.

therapeutic potential. Collectively, these findings indicate that the stem cell secretome is more advantageous than the mature cell secretome in therapeutic application. Furthermore, obtaining a secretome with stimuli could enhance its amount and potential.

Whereas non-specific stimulation has been utilized to obtain the secretome from ASCs to date, the present study first adopted the concept of disease-specific stimulation. First, the term isecretome was created. Isecretome literally refers to 'induced secretome', which indicates a secretome induced by the specific disease-causing agents. The concept of 'pre-sensitization by disease-causing agents' has already been demonstrated. For example, Prado et al proposed a method of treating a disease using EVs obtained by pre-sensitizing mature cells using disease-causing agents (28). They pre-sensitized the mice by respiratory exposure to Ole e 1 (an allergen), and then obtained EVs from bronchoalveolar lavage fluid. Subsequently, the 
A

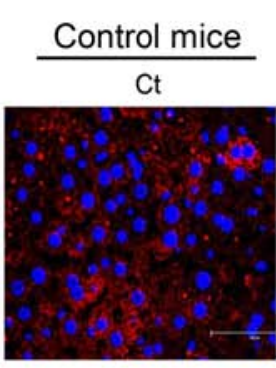

B

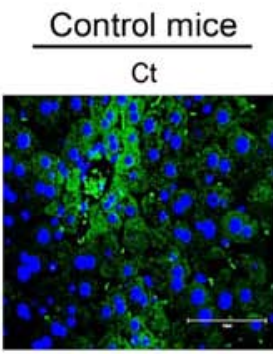

F4/80

Mice with hepatitis B
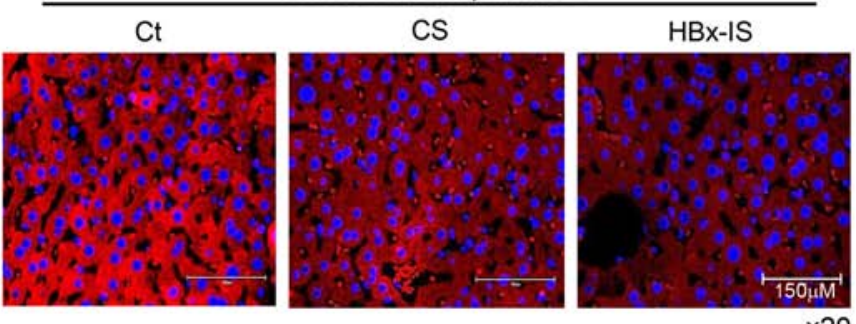

$\times 20$

CD68

Mice with hepatitis B

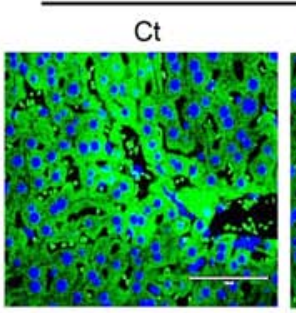

Mice with hepatitis B

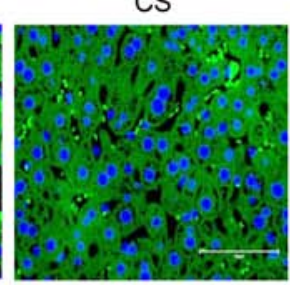

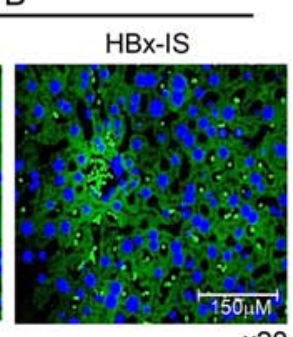

$\times 20$
$\mathrm{F} 4 / 80$

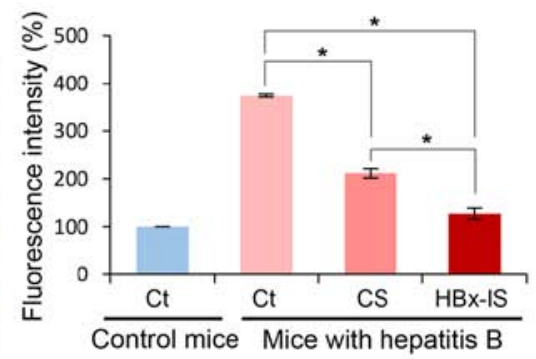

Figure 7. Immunofluorescence of the liver following administration of the HBx-isecretome. (A) Left panel illustrates F4/80 immunofluorescence of the liver following the administration of each treatment. Right panel presents the percentages of F4/80 immunoreactive areas. Injection of CS and HBx-IS both significantly decreased the expression of F4/80 in the liver. In addition, the expression of F4/80 was significantly lower in the HBx-IS group than in the CS group. (B) Left panel illustrates CD68 immunofluorescence of the liver following administration of each treatment. Right panel presents the percentages of CD68 immunoreactive areas. Injection of CS and HBx-IS both significantly decreased the expression of CD68 in the liver. In addition, the expression of CD68 was significantly lower in the HBx-IS group than in the CS group. Values are presented as the means \pm standard deviation of 3 independent experiments. Percentages of immunoreactive areas were measured using $\mathrm{NIH}$ image $\mathrm{J}$ and expressed as relative values to those in normal livers. * $\mathrm{P}<0.05$. HBx, hepatitis B virus protein X; CS, control secretome; HBx-IS, indirect HBx-induced secretome; HBx-DS, direct HBx-induced secretome.

obtained EVs were administrated intranasally to the new mice. It was demonstrated that the mice treated with the EVs did not experience allergy to Ole e 1 , reaching to a tolerant status. This suggests that respiratory tract cells produced protective materials in response to Ole e 1, which prevented allergy by Ole e 1 in new mice. Unlike the research of Prado et al in which mature respiratory cells were utilized, the present study utilized ASCs with a higher responsiveness and plasticity, thus, raising the possibility of their application to a variety of therapeutics.

In the present study, the hypothesis put forth was that the appropriate stimulation of MSCs with pathogenic agents could lead to the production of a secretome specialized for exerting protective effects against the pathogen. In a previous study, the authors fist validated this hypothesis by demonstrating the superiority of the TAA-isecretome in a mouse model of TAA-induced hepatic failure (19). In that study, the authors collected the secretory materials (named as inducers) released from AML12 hepatocytes that had been pre-treated with TAA and generated the TAA-induced secretome (TAA-isecretome) after stimulating the ASCs with the inducers. The TAA-isecretome was intravenously administered to mice with TAA-induced hepatic failure and those with partial hepatectomy. TAA-isecretome infusion exhibited greater therapeutic potential in terms of i) restoring disorganized hepatic tissue to normal tissue; ii) inhibiting pro-inflammatory cytokines (IL-6 and TNF- $\alpha$ ); and iii) reducing abnormally elevated liver enzymes (AST and
ALT) compared to the naïve secretome infusion in mice with TAA-induced hepatic failure. However, the TAA-isecretome exhibited an inferior therapeutic potential for restoring hepatic function in partially hepatectomized mice. Therefore, it was concluded that the appropriate stimulation of MSCs with pathogenic agents may lead to the production of a secretome specialized for protecting against the pathogen.

In order to produce a disease-specific secretome, it is essential to select appropriate pathogens that stimulate MSCs, as well as the precise determination of the reaction condition. It should be highlighted that pathogenic stimuli should not be too weak or too strong as they could lead to a lack of a response or destruction of MSCs, respectively. In order to determine the appropriate isecretome for the mouse model of hepatitis $\mathrm{B}$, the present study deliberately examined a variety of conditions, and compressed the isecretome candidates into HBx-DS and HBx-IS. Subsequently, it was found that HBx-IS exerted more potent anti-inflammatory, liver regenerative, and anti-apoptotic effects in the mouse model of hepatitis B than HBx-DS in the present study. Thus, it was concluded that HBs-IS could be a more acceptable candidate of isecretome for hepatitis B than HBx-DS.

There are still many incurable diseases, most of which are due to the inability of the mature cells of patients to neutralize or inhibit pathogenic agents. However, it should be emphasized that while the mature cells of patients are unable to produce the protective materials against pathogenic agents, MSCs could be able to produce these, as MSCs have superior 

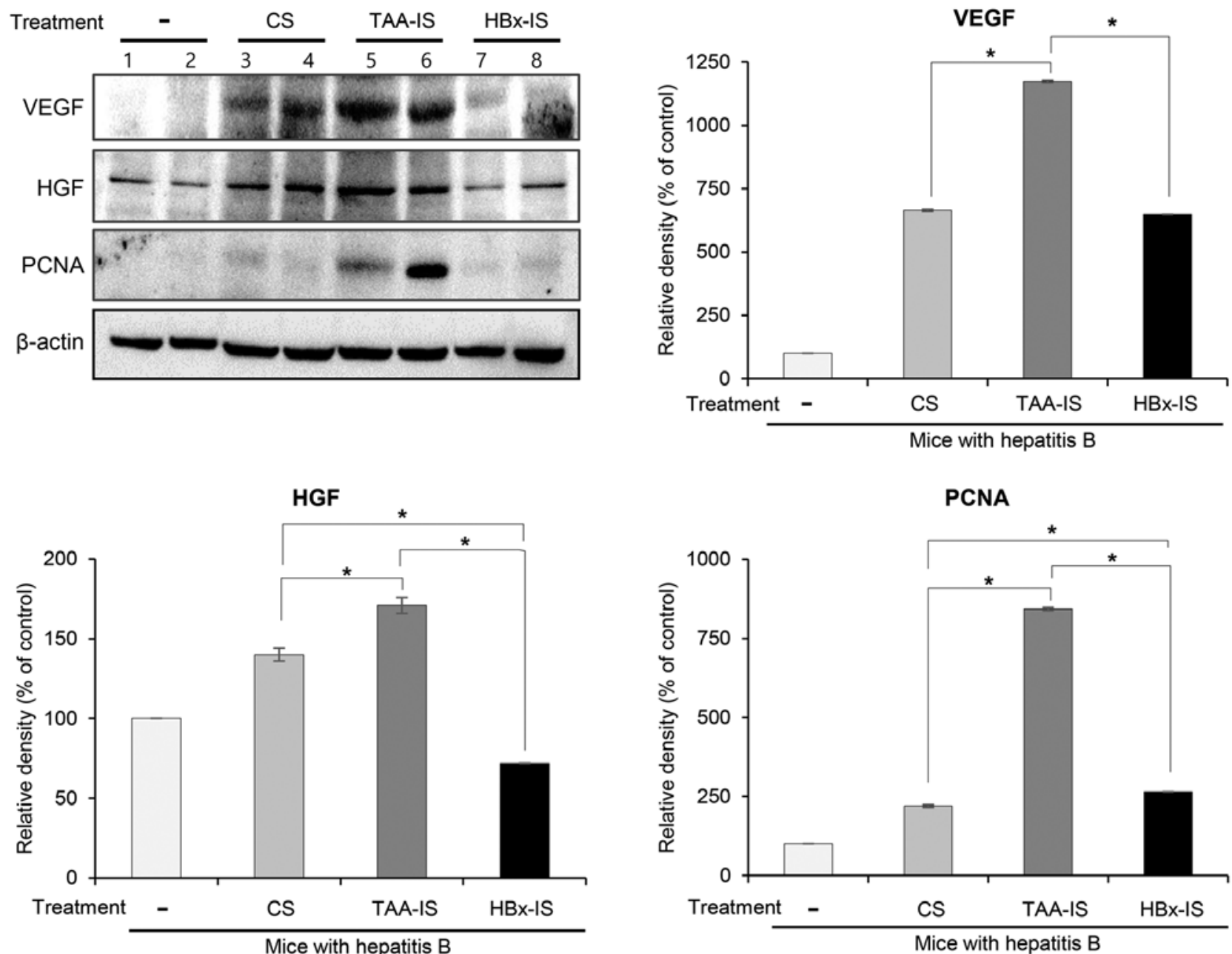

Figure 8. Validation of disease specificity of HBx-isecretome. The mice with TAA-induced hepatic failure were intravenously infused with the control secretome, TAA-isecretome and $\mathrm{HBx}$-isecretome, respectively. Western blot analysis of the livers revealed that TAA-isecretome infusion, rather than $\mathrm{HBx}$-isecretome infusion, induced a higher expression of proliferation markers (HGF, VEGF and PCNA) (all P<0.05). Values are presented as the means \pm standard deviation of 3 independent experiments. " $\mathrm{P}<0.05$. Relative densities of individual markers were quantified using Image Lab software and were then normalized to those of $\beta$-actin in each group. CS, control secretome; $\mathrm{Ct}$, control; $\mathrm{HBx}$, hepatitis B virus protein X; HBx-IS, indirect HBx-induced secretome; HGF, hepatocyte growth factor; PCNA, proliferating cell nuclear antigen; TAA, thioacetamide; TAA-IS, thioacetamide-induced secretome; VEGF, vascular endothelial growth factor.

responsiveness and plasticity than mature cells (29-31). The isecretome produced by MSCs is the collections of protective materials against pathogenic agents most of which mature cells could not produce. It is expected that the application of such an isecretome concept may pave the way for the treatment of several incurable diseases.

HBx is the protein encoded by the HBx gene that is one of four open reading frames comprising the HBV genome. $\mathrm{HBx}$ plays a key role in HBV transcription and replication. This is achieved by the regulation of viral promoters and enhancers by HBx $(32,33)$. Furthermore, HBx is involved in the development of hepatocellular carcinoma, as well as in the regulation of checkpoints in the cell cycle $(34,35)$. In particular, HBx plays a dual role in the regulation of the apoptotic process, which indicates that $\mathrm{HBx}$ inhibits, as well as promotes cellular apoptosis (36). Anti-apoptosis by $\mathrm{HBx}$ is achieved via activating NF- $\mathrm{kB}$, an activator of anti-apoptotic signals (37). On the other hand, HBx exerts pro-apoptotic effects when NF- $\mathrm{kB}$ is inhibited (37). Since HBx exerts various effects on cellular apoptosis, it is particularly difficult to generate appropriate an HBx-isecretome against hepatitis B. The authors aim to perform the component analysis of HBx-IS and HBx-DS, which is expected to clarify the therapeutic mechanism of the HBx-isecretome.

In conclusion, the present study demonstrated a method which may be used to enhance the disease-specific therapeutic effects of the secretome. Specifically, the therapeutic potential of the HBx-isecretome (HBx-IS) in mice with hepatitis B was determined. Compared with the CS injection, the HBx-IS injection more significantly reduced the serum levels of pro-inflammatory cytokines. In addition, the HBx-IS injection led to a higher expression of liver regeneration-related markers, a lower expression of pro-apoptotic markers in mouse livers, and a lower expression of pro-inflammatory markers in the liver compared to the CS injection. These results collectively indicate that the HBx-IS exhibits greater liver regenerative, anti-inflammatory, and anti-apoptotic properties, particularly in mice with hepatitis $B$, than the CS. This suggests that the secretome obtained by stimulating ASCs with the disease-causing agents may exert a more potent therapeutic effect in the specific disease than naïve secretomes. This approach is expected to pave the way to 
the development of novel various specific therapeutics based on the high plasticity and responsiveness of MSCs to disease-causing agents.

\section{Acknowledgements}

Not applicable.

\section{Funding}

No funding was received.

\section{Availability of data and materials}

The datasets used and/or analyzed during the current study are available from the corresponding author on reasonable request.

\section{Authors' contributions}

All authors contributed to manuscript preparation. SJK designed the research, analyzed the data and contributed to manuscript preparation. HJK also analyzed the data and contributed to manuscript preparation. OHK performed the in vitro experiments and also contributed to manuscript preparation. HEH performed various in vitro and in vivo experiments. SCL was involved in designing the study and analyzing data. All authors read and approved the final manuscript.

\section{Ethics approval and consent to participate}

Animal experiments were carried out in compliance with the guidelines of the Institute for Laboratory Animal Research, Korea (IRB no. CMCDJ-AP-2016-001). The Ethics Committee at Daejeon St. Mary's hospital, the Catholic University of Korea, approved the animal experiments for the research (IRB no. CMCDJ-AP-2016-001).

\section{Patient consent for publication}

Not applicable.

\section{Competing interests}

The authors declare that they have no competing interests.

\section{References}

1. Meirelles Lda S, Fontes AM, Covas DT and Caplan AI Mechanisms involved in the therapeutic properties of mesenchymal stem cells. Cytokine Growth Factor Rev 20: 419-427, 2009.

2. An SY, Jang YJ, Lim HJ, Han J, Lee J, Lee G, Park JY, Park SY, Kim JH, Do BR, et al: Milk fat globule-EGF factor 8, secreted by mesenchymal stem cells, protects against liver fibrosis in mice. Gastroenterology 152: 1174-1186, 2017.

3. Makridakis M, Roubelakis MG and Vlahou A: Stem cells: Insights into the secretome. Biochim Biophys Acta 1834: 2380-2384, 2013.

4. Parekkadan B, van Poll D, Suganuma K, Carter EA, Berthiaume F, Tilles AW and Yarmush ML: Mesenchymal stem cell-derived molecules reverse fulminant hepatic failure. PLoS One 2: e941, 2007.
5. Paul G and Anisimov SV: The secretome of mesenchymal stem cells: Potential implications for neuroregeneration. Biochimie 95: 2246-2256, 2013.

6. Salgado AJ, Oliveira JM, Martins A, Teixeira FG, Silva NA, Neves NM, Sousa N and Reis RL: Tissue engineering and regenerative medicine: Past, present, and future. Int Rev Neurobiol 108: 1-33, 2013.

7. Lavoie JR and Rosu-Myles M: Uncovering the secretes of mesenchymal stem cells. Biochimie 95: 2212-2221, 2013.

8. Baglio SR, Pegtel DM and Baldini N: Mesenchymal stem cell secreted vesicles provide novel opportunities in (stem) cell-free therapy. Front Physiol 3: 359, 2012.

9. Rubio D, Garcia S, Paz MF, De la Cueva T, Lopez-Fernandez LA, Lloyd AC, Garcia-Castro J and Bernad A: Molecular characterization of spontaneous mesenchymal stem cell transformation. PLoS One 3: e1398, 2008.

10. Konala VBR, Mamidi MK, Bhonde R, Das AK, Pochampally R and Pal R: The current landscape of the mesenchymal stromal cell secretome: A new paradigm for cell-free regeneration. Cytotherapy 18: 13-24, 2016.

11. Salgado AJ, Sousa JC, Costa BM, Pires AO, Mateus-Pinheiro A, Teixeira FG, Pinto L and Sousa N: Mesenchymal stem cells secretome as a modulator of the neurogenic niche: Basic insights and therapeutic opportunities. Front Cell Neurosci 9: 249, 2015.

12. Waszak P, Alphonse R, Vadivel A, Ionescu L, Eaton F and Thebaud B: Preconditioning enhances the paracrine effect of mesenchymal stem cells in preventing oxygen-induced neonatal lung injury in rats. Stem Cells Dev 21: 2789-2797, 2012.

13. Lee SC, Jeong HJ, Lee SK and Kim SJ: Lipopolysaccharide preconditioning of adipose-derived stem cells improves liver-regenerating activity of the secretome. Stem Cell Res Ther 6: 75, 2015

14. Lee SC, Jeong HJ, Lee SK and Kim SJ: Hypoxic conditioned medium from human adipose-derived stem cells promotes mouse liver regeneration through JAK/STAT3 signaling. Stem Cells Transl Med 5: 816-825, 2016.

15. Lee SC, Kim KH, Kim OH, Lee SK, Hong HE, Won SS, Jeon SJ, Choi BJ, Jeong W and Kim SJ: Determination of optimized oxygen partial pressure to maximize the liver regenerative potential of the secretome obtained from adipose-derived stem cells Stem Cell Res Ther 8: 181, 2017.

16. Noiseux N, Gnecchi M, Lopez-Ilasaca M, Zhang L, Solomon SD, Deb A, Dzau VJ and Pratt RE: Mesenchymal stem cells overexpressing Akt dramatically repair infarcted myocardium and improve cardiac function despite infrequent cellular fusion or differentiation. Mol Ther 14: 840-850, 2006.

17. Wang X, Zhao T, Huang W, Wang T, Qian J, Xu M, Kranias EG, Wang Y and Fan GC: Hsp20-engineered mesenchymal stem cells are resistant to oxidative stress via enhanced activation of Akt and increased secretion of growth factors. Stem Cells 27: 3021-3031, 2009

18. Mohammadzadeh M, Halabian R, Gharehbaghian A, Amirizadeh N, Jahanian-Najafabadi A, Roushandeh AM and Roudkenar MH: Nrf-2 overexpression in mesenchymal stem cells reduces oxidative stress-induced apoptosis and cytotoxicity. Cell Stress Chaperones 17: 553-565, 2012.

19. Kim OH, Hong HE, Seo H, Kwak BJ, Choi HJ, Kim KH, Ahn J, Lee SC and Kim SJ: Generation of induced secretome from adipose-derived stem cells specialized for disease-specific treatment: An experimental mouse model. World J Stem Cells 12: 70-86, 2020.

20. Livak KJ and Schmittgen TD: Analysis of relative gene expression data using real-time quantitative PCR and the 2(T)(-Delta Delta C) method. Methods 25: 402-408, 2001.

21. Li X, Liu G, Chen M, Yang Y, Xie Y and Kong X: A novel hydrodynamic injection mouse model of HBV genotype $\mathrm{C}$ for the study of HBV biology and the anti-viral activity of lamivudine. Hepat Mon 16: e34420, 2016.

22. Ling LR, Zheng DH, Zhang ZY, Xie WH, Huang YH, Chen ZX, Wang XZ and Li D: Effect of HBx on inflammation and mitochondrial oxidative stress in mouse hepatocytes. Oncol Lett 19: 2861-2869, 2020

23. Chierchia A, Chirico N, Boeri L, Raimondi I, Riva GA, Raimondi MT, Tunesi M, Giordano C, Forloni G and Albani D: Secretome released from hydrogel-embedded adipose mesenchymal stem cells protects against the Parkinson's disease related toxin 6-hydroxydopamine. Eur J Pharm Biopharm 121: 113-120, 2017.

24. Driscoll $\mathrm{J}$ and Patel T: The mesenchymal stem cell secretome as an acellular regenerative therapy for liver disease. J Gastroenterol 54: 763-773, 2019. 
25. Eleuteri $\mathrm{S}$ and Fierabracci A: Insights into the secretome of mesenchymal stem cells and its potential applications. Int J Mol Sci 20: 4597, 2019.

26. Li N, Sarojini H, An J and Wang E: Prosaposin in the secretome of marrow stroma-derived neural progenitor cells protects neural cells from apoptotic death. J Neurochem 112: 1527-1538, 2010.

27. Rodríguez-Suárez E, Gonzalez E, Hughes C, Conde-Vancells J, Rudella A, Royo F, Palomo L, Elortza F, Lu SC, Mato JM, et al: Quantitative proteomic analysis of hepatocyte-secreted extracellular vesicles reveals candidate markers for liver toxicity. J Proteomics 103: 227-240, 2014.

28. Prado N, Marazuela EG, Segura E, Fernández-García H, Villalba M, Théry C, Rodriguez R and Batanero E: Exosomes from bronchoalveolar fluid of tolerized mice prevent allergic reaction. J Immunol 181: 1519-1525, 2008.

29. Bobis S, Jarocha D and Majka M: Mesenchymal stem cells: Characteristics and clinical applications. Folia Histochem Cytobiol 44: 215-230, 2006.

30. Filip S, Mokrý J, English D and Vojácek J: Stem cell plasticity and issues of stem cell therapy. Folia Biol (Praha) 51: 180-187, 2005.

31. Frisén J: Stem cell plasticity? Neuron 35: 415-418, 2002.

32. Chen HS, Kaneko S, Girones R, Anderson RW, Hornbuckle WE, Tennant BC, Cote PJ, Gerin JL, Purcell RH and Miller RH: The woodchuck hepatitis virus X gene is important for establishment of virus infection in woodchucks. J Virol 67: 1218-1226, 1993.
33. Zoulim F, Saputelli J and Seeger C: Woodchuck hepatitis virus $\mathrm{X}$ protein is required for viral infection in vivo. J Virol 68: 2026-2030, 1994

34. Choe BH: Hepatitis B virus: Pathogenesis, molecular diagnosis, and clinical significance of mutation. Korean J Pediatr Gastroenterol Nutr 10: 51-65, 2007.

35. Neuveut C, Wei Y and Buendia MA: Mechanisms of HBV-related hepatocarcinogenesis. J Hepatol 52: 594-604, 2010.

36. Slagle BL and Bouchard MJ: Role of $\mathrm{HBx}$ in hepatitis B virus persistence and its therapeutic implications. Curr Opin Virol 30: 32-38, 2018

37. Clippinger AJ, Gearhart TL and Bouchard MJ: Hepatitis B virus $\mathrm{X}$ protein modulates apoptosis in primary rat hepatocytes by regulating both NF-kappaB and the mitochondrial permeability transition pore. J Virol 83: 4718-4731, 2009.

(i) $(9)$ This work is licensed under a Creative Commons Attribution-NonCommercial-NoDerivatives 4.0 International (CC BY-NC-ND 4.0) License. 